\title{
On Equivalence of Duffin-Kemmer-Petiau and Klein-Gordon Equations
}

\author{
V. Ya. Fainberg* and B. M. Pimentel \\ Instituto de Física Teórica, \\ Universidade Estadual Paulista, \\ Rua Pamplona 145, São Paulo, S.P, 01405-900, Brazil. \\ E-mails: fainberg@ift.unesp.br, pimentel@ift.unesp.br
}

Received 7 January, 2000

\begin{abstract}
A strict proof of equivalence between Duffin-Kemmer-Petiau (DKP) and Klein-Gordon (KG) theories is presented for physical S-matrix elements in the case of charged scalar particles interacting in minimal way with an external or quantized electromagnetic field. First, Hamiltonian canonical approach to DKP theory is developed in matrix form. The theory is then quantized through the construction of the generating functional for Green functions (GF) and the physical matrix elements of S-matrix are proved to be relativistic invariants. The equivalence between both theories is then proved using the connection between GF and the elements of S-matrix in reduction formulas of Lehmann, Symanzik, Zimmermann.
\end{abstract}

\section{Introduction}

More than 60 years ago G. Petiau [1], R Duffin [2] and N. Kemmer [3] proposed the first order equation (DKP equation) for description of spin 0 and 1 particles. This period of time is conventionally divided in three periods: the first one from 1939 until, approximately 1970; the second from 1970 to 1980 and the last one from 1980 on. During the first period the majority of the papers about DKP equation was devoted to the development of DKP formalism and to the investigation of DKP charged particles interaction with electromagnetic field (EM field). For many classes of processes (such as QE of spin 0 mesons, meso-atom and others) calculations based on DKP and KG equations yield identical results [5], including one-loop corrections $[4,5,6]^{1}$.

The second period can be characterized as by some disappointments and hesitations. By this time two great discoveries had been made: parity violation and creation of unified theory of electro-weak interaction (Weinberg-Salam theory or Standard Model). The question about the equivalence of both DKP and KG theories arises again at the attempts to describe new processes. Many works (see references in [8]) have been made applying DKP formalism to decays of $K$ and other unstable mesons and to strong interaction. The conclusion presented in reference [8] was not optimistic: DKP formalism in some cases yield different results from a second order formalism ${ }^{2}$. The third period goes under the sign of uncertainness: are both DKP and KG equivalent or not? Not so many papers have been published on this theme. In our opinion one of main reasons for the decrease of interest in DKP formalism in the last period is the conclusion about nonequivalence between DKP and KG theories ${ }^{3}$. We believe that the equivalence between these theories in the case of nonstable particles can be proved as well as for all processes which are described by renormalizable theories. This question, however, goes beyond the scope of this paper. As we know there are no strict proof of the equivalence between DKP and KG theories in Quantum Electrodynamics of spin 0 particles, too. Coincident results have been obtained for many processes in first order pertubation theory, one loop corrections and the infrared approximation $[5,6,7]$. The main goal of

\footnotetext{
* Permanent address: P. N. Lebedev Institute of Physics, Moscow, Russia.

${ }^{1} \mathrm{~A}$ rich list of references with historical comments can be found in reference [8]. Unfortunately in this work there are no references to the works by I. Gelfand and A. Yaglom, who obtained the first order equation for particles with fixed arbitrary spin. For references to these and others works see [9].

${ }^{2}$ Moreover, in work [10] it is affirmed that DKP theory gives for $K$ meson decay qualitatively different results when compared to KG-formalism

${ }^{3}$ See section V; Conclusion, point 5 .
} 
this paper is to give a strict proof of the equivalence of DKP and KG theories for charged scalar particles interacting with an external or quantized EM field in minimal way for physical matrix elements in any order of pertubation theory. In section II Hamiltonian canonical approach to DKP theory is developed in matrix form. The construction of generating functional of Green function (GF) of DKP theory is used for quantization of the theory and the physical matrix elements of S-matrix are proved to be relativistic invariants. In section III the equivalence of DKP and KG theories is proved for physical matrix elements utilizing the connection between GF and the elements of S-matrix, including the case of many photons states. For the proof we use the reduction formulas of Lehmann, Symanzik, Zimmermann [11].

In section IV we shortly discuss the basic results and questions about construction of renormalizable DKP theory for spin 0 particles.

\section{Canonical Quantization}

\section{II.1 Hamiltonian approach in matrix form}

Our aim is to construct the Hamiltonian for DKP theory which is one with constraints due to degeneration of $\beta$ matrices. The Lagrangian density is

$$
\mathcal{L}=\bar{\psi}\left(i \beta_{\mu} D^{\mu}-m\right) \psi
$$

where $D^{\mu}=\partial^{\mu}-i e A^{\mu} ; \partial^{\mu}=\frac{\partial}{\partial x_{\mu}} ; g_{\mu \nu}=$ $\operatorname{diag}\{1,-1,-1,-1\}$. Primarily one considers $A^{\mu}$ as an external EM field. We choose the $\beta_{\mu}$ matrices in the following form:

$$
\begin{aligned}
& \beta_{0}=\left|\begin{array}{ccccc}
0 & i & 0 & 0 & 0 \\
-i & 0 & 0 & 0 & 0 \\
0 & 0 & 0 & 0 & 0 \\
0 & 0 & 0 & 0 & 0 \\
0 & 0 & 0 & 0 & 0
\end{array}\right| \\
& \beta_{1}=\left|\begin{array}{ccccc}
0 & 0 & -i & 0 & 0 \\
0 & 0 & 0 & 0 & 0 \\
-i & 0 & 0 & 0 & 0 \\
0 & 0 & 0 & 0 & 0 \\
0 & 0 & 0 & 0 & 0
\end{array}\right| \\
& \beta_{2}=\left|\begin{array}{ccccc}
0 & 0 & 0 & -i & 0 \\
0 & 0 & 0 & 0 & 0 \\
0 & 0 & 0 & 0 & 0 \\
-i & 0 & 0 & 0 & 0 \\
0 & 0 & 0 & 0 & 0
\end{array}\right|
\end{aligned}
$$

$$
\begin{aligned}
\beta_{3} & =\left|\begin{array}{ccccc}
0 & 0 & 0 & 0 & -i \\
0 & 0 & 0 & 0 & 0 \\
0 & 0 & 0 & 0 & 0 \\
0 & 0 & 0 & 0 & 0 \\
-i & 0 & 0 & 0 & 0
\end{array}\right| \\
\bar{\psi}_{\alpha} & =\left(\psi^{*} \eta\right)_{\alpha} \\
& =\left(\varphi^{*}, \varphi^{* 0},-\varphi^{* 1},-\varphi^{* 2},-\varphi^{* 3}\right) ; \\
\eta & =2\left(\beta_{0}\right)^{2}-1 ; \\
\psi_{\alpha} & =\left(\varphi, \varphi^{0}, \varphi^{1}, \varphi^{2}, \varphi^{3}\right) .
\end{aligned}
$$

Starting from equation (1) we can define the momenta

$$
\begin{gathered}
p_{\alpha}=\frac{\partial \mathcal{L}}{\partial . \psi_{\alpha}}=i\left(\psi^{*} \beta_{0}\right)_{\alpha}, p_{\alpha}^{*}=\frac{\partial \mathcal{L}}{\partial . \psi_{\alpha}^{*}}=0, \\
p^{\alpha}=\frac{\partial \mathcal{L}}{\partial . \psi_{\alpha}^{\alpha}}=0, p^{* \alpha}=\frac{\partial \mathcal{L}}{\partial . \psi_{\alpha}^{*}}=0,
\end{gathered}
$$

for $\alpha=1,2,3 \equiv i$. The initial Hamiltonian $H$ is equal to:

$$
\begin{aligned}
H & =\int d^{3} x\left\{p^{\alpha} . \psi_{\alpha}+p^{* \alpha} . \psi_{\alpha}^{*}-\mathcal{L}\right\} \\
& =\int d^{3} x\left(-i \bar{\psi} \beta_{k} D^{k} \psi+\bar{\psi} m \psi-e \bar{\psi} \beta_{0} A^{0} \psi\right) \\
& =\int d^{3} x\left(-i \bar{\psi} \beta_{k} \partial^{k} \psi+m \bar{\psi} \psi-e \bar{\psi} \beta_{\mu} A^{\mu} \psi\right),(6)
\end{aligned}
$$

where $k=1,2,3$. Here we write down all the $1^{\text {st }}$ stage and the $2^{\text {st }}$ stage constraints and Lagrangian multipliers in matrix form, omitting calculations: $-1^{\text {st }}$ stage constraints 4

$$
\begin{aligned}
\theta_{\alpha} & =p_{\alpha}-i\left(\bar{\psi} \beta_{0}\right)_{\alpha} \\
\theta_{\alpha}^{*} & =p_{\alpha}^{*}
\end{aligned}
$$

- $2^{\text {nd }}$ stage constraints

$$
\left.\begin{array}{rl}
\theta_{2}^{\alpha} & =\left[\left(1-\left(\beta_{0}\right)^{2}\right)\left(i \beta_{k} D^{k}-m\right) \psi\right]^{\alpha} \\
\theta_{2}^{* \alpha} & =\left[\bar{\psi}\left(i \beta_{k}{\overleftarrow{D^{*}}}^{k}+m\right)\left(1-\left(\beta_{0}\right)^{2}\right)\right]^{\alpha}
\end{array}\right\}
$$

- Lagrangian multipliers

$$
\begin{gathered}
\left(\beta_{0}^{2} \lambda\right)_{\alpha}=\left[i \beta_{0}\left(i \beta_{k} D^{k}-m\right) \psi\right]_{\alpha} ; \\
\left(\lambda^{*} \beta_{0}^{2}\right)_{\alpha}=-\left[\bar{\psi}\left(i \beta_{k} \overleftarrow{D}^{* k}+m\right) \beta_{0}\right]_{\alpha} ; \quad(10) \\
{\left[\left(1-\left(\beta_{0}\right)^{2}\right)\left(m-i \beta_{k} D^{k}\right) \eta\right]_{\alpha \beta} \lambda^{\beta}=0} \\
\lambda^{* \beta}\left[\eta\left(i \beta_{k} \overleftarrow{D}^{* k}+m\right)\left(1-\left(\beta_{0}\right)^{2}\right)\right]_{\beta \alpha}=0 .(11
\end{gathered}
$$

${ }^{4}$ We follow the terminology of the book [12]. Quantization of theories with the $2^{\text {nd }}$ - class constraints devoted many papers, beginning with the classical work of Dirac [14], see also $[15,16]$. 
For generating functional in external EM field we get the following expression [13]

$$
\begin{aligned}
& \mathcal{Z}(I,-I)=\mathcal{Z}_{0}^{-1} \int \mathcal{D} \psi \mathcal{D} \bar{\psi} \times \\
& \delta\left(\left(1-\left(\beta_{0}\right)^{2}\right)\left(i \beta_{k} D^{k}-m\right) \psi\right) \\
& \times \delta\left(\bar{\psi}\left(i \beta_{k} \overleftarrow{D}^{*}+m\right)\left(1-\left(\beta_{0}\right)^{2}\right)\right) \\
& \times \exp \left\{i \int d ^ { 4 } x \left(\bar{\psi}\left(i \beta_{\mu} D^{\mu}-m\right) \psi\right.\right. \\
& \left.\left.+{ }_{-} I \psi+\bar{\psi} I\right)\right\} .
\end{aligned}
$$

Introducing the auxiliary fields $C$ and $\_C$ instead functional $\delta$ - function in equation (12) we get

$$
\begin{aligned}
\mathcal{Z}\left(I,{ }_{-} I\right)= & \mathcal{Z}_{0}^{-1} \int \mathcal{D} \psi \mathcal{D} \bar{\psi} \mathcal{D} C \mathcal{D}{ }_{-} C \times \\
& \exp \left\{i \int d ^ { 4 } x \left(\bar{\psi}\left(i \beta_{\mu} D^{\mu}-m\right) \psi\right.\right. \\
+ & C\left(1-\left(\beta_{0}\right)^{2}\right)\left(i \beta_{\mu} D^{\mu}-m\right) \psi \\
& \bar{\psi}\left(i \beta_{\mu} \overleftarrow{D}^{\mu}+m\right)\left(1-\left(\beta_{0}\right)^{2}\right) C+ \\
& I \psi+\bar{\psi} I)\}
\end{aligned}
$$

where one used that $\beta_{0}\left(1-\left(\beta_{0}\right)^{2}\right)=0$. Integrating over all fields $\psi, \bar{\psi}, C$ and $\_$we finally obtain:

$$
\begin{aligned}
\mathcal{Z}(I,-I) & =\exp \left\{-i \int d^{4} x d^{4} y I(x)(S(x, y, A)\right. \\
+ & \left.\left.\frac{1}{m}\left(1-\left(\beta_{0}\right)^{2}\right) \delta^{4}(x-y)\right) I(y)\right\}
\end{aligned}
$$

where we have introduced the total GF of DK particle in external EM field $A_{\mu}$ :

$$
S(x, y, A)=\left(i \beta_{\mu} D^{\mu}-m\right)^{-1} \delta^{4}(x-y)
$$

One can make some important comments about expression (15). 1) When we integrate over $\psi, \bar{\psi}, C$ and $\_$divergences appear and infinite expression for $\operatorname{det}\left(i \beta_{\mu} D^{\mu}-m\right)^{-1}$. All this multipliers also arise in $\mathcal{Z}_{0}$ and disappear from the final equation (14) . 2) The nonrelativistic invariant term $\sim\left(1-\left(\beta_{0}\right)^{2}\right)$ in equation (14) arises at excluding nonphysical component $\psi$, due to the second stage constraints $\left(\theta_{i}^{2}, \theta_{i}^{* 2}\right.$ in equation (9)). One can show that this term (which does not depend on charge) does not contribute to physical matrix elements of $S$-matrix [13]. 3)If one generalizes equations (12) to (15) to the case of interaction of DK particles with quantized EM fields we get the following expression for generating functional for all GF of the theory (in $\alpha$-gauge):

$$
\begin{aligned}
\mathcal{Z}(I, & \left.I, \mathcal{J}_{\mu}\right)=\mathcal{Z}_{0}^{-1} \int \mathcal{D} A_{\mu} \exp \left\{-i \int d^{4} x \times\right. \\
& \left(\operatorname{Tr} \ln \frac{S(x, x, A)}{S(x, x, 0)}-\frac{1}{4} F_{\mu \nu} F^{\mu \nu}\right. \\
& -\mathcal{J}_{\mu} A^{\mu}-\frac{1}{2 \alpha}\left(\partial_{\mu} A^{\mu}\right)^{2} \\
& -\int d^{4} y-I(x)(S(x, y, A) \\
& \left.\left.\left.+\frac{1}{m}\left(1-\left(\beta_{0}\right)^{2}\right) \delta^{4}(x-y)\right) I(y)\right)\right\}
\end{aligned}
$$

Here we insert in denominator and in $\mathcal{Z}_{0}$ infinity constant $(\operatorname{det} S(x, x, 0))^{-1}$. As it is well known, the term $\sim \operatorname{Tr} \ln S(x, x, A)$ in equation (16) is responsible for appearance of all vacuum polarizations diagrams. 4) Starting from (16) it is easy to prove that many photons GF coincide in DKP and KG theories. The generating functional of GF in KG theory has the form:

$$
\begin{aligned}
\mathcal{Z}\left(\mathcal{J}^{*},\right. & \left.\mathcal{J}, \mathcal{J}_{\mu}\right)=\mathcal{Z}_{0}^{-1} \int \mathcal{D} A_{\mu} \exp \left\{-i \int d^{4} x \times\right. \\
& \operatorname{Tr}\left(\ln \frac{G(x, x, A)}{G(x, x, 0)}-\frac{1}{4} F_{\mu \nu} F^{\mu \nu}\right. \\
& -\frac{1}{2 \alpha}\left(\partial_{\mu} A^{\mu}\right)^{2}+\mathcal{J}_{\mu} A^{\mu} \\
& \left.\left.-\int d^{4} y \mathcal{J}^{*}(x) G(x, y, A) \mathcal{J}(y)\right)\right\}
\end{aligned}
$$

Here

$$
G(x, y)=\left(D_{\mu} D^{\mu}+m^{2}\right)^{-1} \delta^{4}(x-y)
$$

To get the generating functional of GF only for photons we have to put $\mathcal{J}^{*}=\mathcal{J}=0$ in equation (17) and $I=I=0$ in equation (16). Equality of these equations will be established if we prove that

$$
\begin{aligned}
\mathcal{Z}_{A} & \equiv \operatorname{det} \frac{S(x, y, A)}{S(x, y, 0)}=\exp \operatorname{Tr} \ln \frac{S(x, x, A)}{S(x, x, 0)} \\
& =\operatorname{det} \frac{G(x, y, A)}{G(x, y, 0)} .
\end{aligned}
$$

On the other hand

$$
\begin{aligned}
\mathcal{Z}_{A}= & \mathcal{Z}_{0}^{-1} \int \mathcal{D} \psi \mathcal{D} \bar{\psi} \times \\
& \exp \left\{i \int d^{4} x \bar{\psi}(i \wedge D-m) \psi\right\},
\end{aligned}
$$

where

$$
\mathcal{Z}_{0}=\int \mathcal{D} \psi \mathcal{D} \bar{\psi} \exp \left\{i \int d^{4} x \bar{\psi}(i \wedge \partial-m) \psi\right\}
$$


In component form expression (20) equals to:

$$
\begin{aligned}
\mathcal{Z}_{A}= & \mathcal{Z}_{0}^{-1} \int \mathcal{D} \varphi \mathcal{D} \varphi^{*} \mathcal{D} \varphi_{\mu} \mathcal{D} \varphi_{\mu}^{*} \times \\
& \exp \left\{i \int d ^ { 4 } x \left(\varphi^{* \mu} D_{\mu} \varphi-\varphi^{*} D_{\mu} \varphi^{\mu}\right.\right. \\
& \left.\left.-m\left(\varphi^{*} \varphi+\varphi^{* \mu} \varphi_{\mu}\right)\right)\right\}
\end{aligned}
$$

After integration over $\varphi_{\mu}^{*}$ and $\varphi^{\mu}$ we get

$$
\begin{aligned}
& \mathcal{Z}_{A}=\widetilde{\mathcal{Z}}_{0}^{-1} \int \mathcal{D} \varphi \mathcal{D} \varphi^{*} \times \\
& \exp \left\{-\frac{i}{m} \int d^{4} x \varphi^{*}\left(D^{\mu} D_{\mu}+m^{2}\right) \varphi\right\},
\end{aligned}
$$

where now

$$
\begin{aligned}
& \widetilde{\mathcal{Z}}_{0}^{-1}=\int \mathcal{D} \varphi \mathcal{D} \varphi^{*} \times \\
& \exp \left\{-\frac{i}{m} \int d^{4} x \varphi^{*}\left(\partial^{\mu} \partial_{\mu}+m^{2}\right) \varphi\right\} .
\end{aligned}
$$

Doing substitution the $\frac{\varphi}{\sqrt{m}} \rightarrow \varphi$ we see that the determinant (22) is equal to the right hand side of equation (19). The equivalence was proved.

\section{Equivalence between phys- ical matrix elements of S- matrix in DKP and KG equations}

We use LSZ reduction formulas [11] for proof of the equivalence between both theories. The main goal of LSZ approach is to express the matrix elements of Smatrix through total many particles GF, making minimal assumptions as possible. To apply this approach to DKP theory we must write down the operator's solutions of the free DKP equations. Taking into account that in DKP theory there are only two linearly independent solutions [5] of the free equation one can write

$$
\begin{aligned}
\wedge \psi_{\text {in }, \text { out }}= & \frac{1}{(2 \pi)^{3 / 2}} \int d^{3} p \times \\
& \left\{u^{-}(\mathbf{p}) \wedge a_{\text {in }, \text { out }}^{-}(\mathbf{p}) e^{-i p x}\right. \\
& \left.+u^{+}(\mathbf{p}) \wedge b_{\text {in }, \text { out }}^{+}(\mathbf{p}) e^{i p x}\right\},
\end{aligned}
$$

and

$$
\wedge \bar{\psi}_{\text {in }, \text { out }}=\frac{1}{(2 \pi)^{3 / 2}} \int d^{3} p \times
$$

$$
\begin{aligned}
& \left\{\bar{u}^{+}(\mathbf{p}) \wedge a_{\text {in }, \text { out }}^{+}(\mathbf{p}) e^{i p x}\right. \\
& \left.+\bar{u}^{-}(\mathbf{p}) \wedge b_{\text {in }, \text { out }}^{-}(\mathbf{p}) e^{-i p x}\right\} .
\end{aligned}
$$

Here

$$
\begin{aligned}
& (\wedge p \pm m) u^{ \pm}(\mathbf{p})=0 ; \bar{u}^{\mp}(\mathbf{p})(\wedge p \pm m)=0=0 \\
& \widehat{p} \equiv \beta_{\mu} p^{\mu}, p_{0}=\left(\mathbf{p}^{2}+m^{2}\right)^{1 / 2}=\omega(\mathbf{p})=\omega ;
\end{aligned}
$$

operators $a_{\text {in }, \text { out }}^{ \pm}$and $b_{\text {in,out }}^{ \pm}$satisfy to the usual commutation relations; and the solutions in component form are

$$
u_{\alpha}^{ \pm}=\sqrt{\frac{m}{2 \omega}}\left(1, \pm \frac{i \omega}{m}, \pm \frac{i p^{1}}{m}, \pm \frac{i p^{2}}{m}, \pm \frac{i p^{3}}{m}\right) .
$$

It is easy to check that the scalar products are

$$
\begin{aligned}
u^{* \mp}(\mathbf{p}) \beta_{0} u^{\mp}(\mathbf{p}) & =\bar{u}^{* \mp}(\mathbf{p}) \beta_{0} \bar{u}^{\mp}(\mathbf{p})= \pm 1 \\
\bar{u}^{\mp}(\mathbf{p}) \beta_{0} u^{\mp}(\mathbf{p}) & =0 .
\end{aligned}
$$

the operators $a_{\text {in,out }}^{ \pm}$and $b_{\text {in,out }}^{ \pm}$are those of creation and annihilation with positive and negative charges accordingly. From equations (24) and (25) one also have

$$
\begin{gathered}
\wedge a_{\text {in }, \text { out }}^{-}(\mathbf{p})=\int d^{3} x e^{-i p x} \bar{u}^{-}(\mathbf{p}) \beta_{0} \wedge \psi_{\text {in }, \text { out }}(x), \\
\wedge b_{\text {in out }}^{+}(\mathbf{p})=\int d^{3} x e^{i p x} \bar{u}^{+}(\mathbf{p}) \beta_{0} \wedge \psi_{\text {in }, \text { out }}(x),
\end{gathered}
$$

and

$$
\begin{aligned}
& a_{\text {in }, \text { out }}^{+}(\mathbf{p})=\left(a_{\text {in }, \text { out }}^{-}(\mathbf{p})\right)^{*} ; \\
& b_{\text {in }, \text { out }}^{-}(\mathbf{p})\left(b_{\text {in }, \text { out }}^{+}(\mathbf{p})\right)^{*} .
\end{aligned}
$$

Vacuum and S-matrix are defined as usual as

$$
\begin{aligned}
a_{\text {in }, \text { out }}^{-}(\mathbf{p})|0\rangle_{\text {in }, \text { out }} & =b_{\text {in }, \text { out }}^{-}(\mathbf{p})|0\rangle_{\text {in }, \text { out }}=0 \\
S|0\rangle_{\text {in }, \text { out }} & =|0\rangle_{\text {in }, \text { out }} \\
a_{\text {out }}^{+} & =a_{\text {in }}^{+} S .
\end{aligned}
$$

For any physical matrix element of S-matrix one has

$$
\left.\left\langle n^{\prime}, m^{\prime} ; \text { out }\right| n, m ; \text { in }\right\rangle=\left\langle n^{\prime}, m^{\prime} ; \text { in }|S| n, m ; \text { in }\right\rangle,
$$

where: $n+m=n^{\prime}+m^{\prime}$ is the conservation of charge and

$$
\left|n, m ; ;_{\text {out }}^{\text {in }}\right\rangle=i=1 n \sqcap j=1 m \sqcap a \text { inout }{ }^{+}\left(\mathbf{p}_{i}\right) b \text { inout }{ }^{+}\left(\mathbf{q}_{j}\right)|0\rangle .
$$


Now we can formulate the main assumption of LSZ approach ${ }^{5}$. For any matrix elements of Heisenberg operators $\wedge \psi(x)$ and $\wedge \bar{\psi}(x)$ the following asymptotic relations are implemented

$$
\begin{aligned}
& x_{0} \rightarrow \mp \infty \lim \left\langle n^{\prime}, m^{\prime} ;_{\text {out }}^{\text {in }}|\wedge \psi(x)| n, m_{\text {out }}^{\text {in }}\right\rangle= \\
& =\left\langle n^{\prime}, m^{\prime} ;_{\text {out }}^{\text {in }}\right| \wedge \psi \text { in } \\
& \text { out }(x)\left|n, m ;_{\text {out }}^{\text {in }}\right\rangle
\end{aligned}
$$

and the same relation for $\wedge \bar{\psi}(x)$. In order to do not complicate the proof of the equivalence between DKP and KGF theories we are restricted by consideration of the matrix elements of S-matrix for particles with the same (positive) charges and one utilizes LSZ reduction formula. We have

$$
\begin{aligned}
& \left\langle 0\left|i=1 k \sqcap a_{\text {out }}^{-}\left(\mathbf{p}_{i}\right) j=1 k \sqcap a_{\text {in }}^{+}\left(\mathbf{q}_{j}\right)\right| 0\right\rangle= \\
= & \left\langle 0\left|i=1 k-1 \sqcap a_{\text {out }}^{-}\left(\mathbf{p}_{i}\right) a_{\text {out }}^{-}\left(\mathbf{p}_{k}\right) j=1 k \sqcap a_{\text {in }}^{+}\left(\mathbf{q}_{j}\right)\right| 0\right\rangle \\
= & c\left\langle n_{1}, \ldots, n_{k-1} ; \text { out }\right| \times \\
& \int d^{4} x e^{i p x} \bar{u}^{-}(\mathbf{p})\left(i \beta_{\mu} \vec{\partial}_{x}^{\mu}-m\right) \wedge \psi(x) \times \\
& \left.\mid m_{1}, \ldots, m_{k} ; \text { in }\right\rangle,
\end{aligned}
$$

where $p_{0}=\omega(\mathbf{p})$ and $c$ is a not essential constant. Utilizing equations (3) and (27) we can rewrite equation (38) in component form

$$
\begin{aligned}
& c\left\langle n_{1}, \ldots, n_{k-1} ; \text { out }\right| \times \\
& \left\{-\frac{1}{m} e^{i p x}\left(\vec{\square}_{x}^{\mu}+m^{2}\right) \wedge \varphi(x)+\right. \\
& \left.+\frac{\partial}{\partial x^{\mu}}\left[e^{i p x}\left(\frac{1}{m} \partial_{x}^{\mu} \wedge \varphi(x)-\wedge \varphi^{\mu}(x)\right)\right]\right\} \times . \\
& \left.\mid n_{1}, \ldots, n_{k} ; \text { in }\right\rangle
\end{aligned}
$$

The main idea of the proof is to show that the second term under total derivative in equation (39) is equal zero. We have for the second term, omitting $|i n\rangle$ and $\langle$ out $|$ states,

$$
\begin{aligned}
& \int d^{4} x \frac{\partial}{\partial x^{\mu}}\left[e^{i p x}\left(\frac{1}{m} \partial_{x}^{\mu} \wedge \varphi(x)-\wedge \varphi^{\mu}(x)\right)\right]= \\
= & \int d \sigma_{\mu} e^{i p x}\left(\frac{1}{m} \partial_{x}^{\mu} \wedge \varphi(x)-\wedge \varphi^{\mu}(x)\right) .
\end{aligned}
$$

One can choose the surface $\sigma_{\mu}$ so that

$$
\sigma_{\mu}:\left\{-T \leq x_{0} \leq T ;-L \leq x_{i} \leq L, \subset=1,2,3\right\} .
$$

The first term, $\mu=0$, equals

$$
\begin{aligned}
& \int d^{3} x\left[e ^ { i \omega T + i \mathbf { p x } } \left(\frac{1}{m} \frac{\partial}{\partial T} \wedge \varphi(\mathbf{x}, T)\right.\right. \\
& \left.-\wedge \varphi^{0}(\mathbf{x}, T)\right)-e^{-i \omega T+i \mathbf{p x}}\left(\frac{1}{m} \frac{\partial}{\partial T} \wedge \varphi(\mathbf{x},-T)\right. \\
& \left.\left.-\wedge \varphi^{0}(\mathbf{x},-T)\right)\right] .
\end{aligned}
$$

Since

$$
\begin{aligned}
T \rightarrow \infty \lim \frac{\partial}{\partial T} \wedge \varphi(\mathbf{x}, \pm T) & =\frac{\partial}{\partial T} \wedge \varphi \text { out } \\
\operatorname{in}(\mathbf{x}, \pm T) & =\wedge \varphi \text { out } \\
\operatorname{in}^{0}(\mathbf{x}, \pm T) & =
\end{aligned}
$$

the first term in (40) disappears in the limit $T \rightarrow \pm \infty$. More delicate situation arises at the proof of disappearance of contribution of terms $d \sigma_{i}$ (see equation (41)) when $L \rightarrow \pm \infty$ at fixed $T$. Mathematically strictly it is possible to do proves only introducing the wave packets instead of plane waves with given momentum. We do not have a place to go in details of the proof. If one introduces total set of orthonormalizing packets $f_{n}(\mathbf{p})$ the contribution will be equal to

$$
\begin{array}{r}
I_{1}(L) \equiv \int d x_{0} d x_{\perp} \int d \mathbf{p} f_{n}(\mathbf{p}) e^{i \omega x_{0}-i p_{\perp} x_{\perp}} \times \\
\left\{e^{-i p_{1} x_{1}}\left(\frac{1}{m} \partial_{x}^{1} \wedge \varphi(x)-\wedge \varphi^{1}(x)\right)_{x_{1}=L_{1}}-\right. \\
\left.-e^{-i p_{1} x_{1}}\left(\frac{1}{m} \partial_{x}^{1} \wedge \varphi(x)-\wedge \varphi^{1}(x)\right)_{x_{1}=-L_{1}}\right\}
\end{array}
$$

where $p_{\perp}=\left(p_{1}, p_{2}\right), x_{\perp}=\left(x_{1}, x_{2}\right)$ and $f_{n}(\mathbf{p})$ is a wave packet. For Gaussian type of packets

$$
f_{n}(\mathbf{p})=\exp \left[\left(-\frac{\mathbf{p}^{2}}{2 m^{2}}\right) i=13 \sqcap H_{n}\left(\frac{p_{i}}{m}\right)\right],
$$

\footnotetext{
${ }^{5}$ This assumption can be proved in the case of microcausality theories, when commutator $[\wedge \psi(x), \wedge \bar{\psi}(y)]=0,(x-y)^{2}<0$.
} 
where $H_{n}(x)$ denotes a Hermite polynomial of degree $n$, it is possible to prove that $I_{1}(L)$ decreases $\sim \exp \left(-(L m)^{2}\right)$. Thus, all the $I_{i}(L), i=1,2,3$ are equal zero in limit $L \rightarrow \pm \infty$. If we repeat the LSZ procedure for the second operators $a_{\text {out }}^{+}\left(\mathbf{p}_{2}\right)$ in equation (38) we get

$$
\begin{aligned}
& \langle k ; \text { in }| k ; \text { out }\rangle=c\langle k-2 ; \text { in }| \int d^{4} x_{1} d^{4} x_{2} \times \\
& e^{i p_{1} x_{1}}\left(\frac{\vec{\square}_{1}+m^{2}}{m}\right)\left\{e^{i p_{2} x_{2}}\left(\frac{\vec{\square}_{2}+m^{2}}{m}\right)\right. \\
& T\left(\wedge \varphi\left(x_{1}\right) \wedge \varphi\left(x_{2}\right)\right)+ \\
& +\partial_{\mu}^{x_{2}}\left[e ^ { i p _ { 2 } x _ { 2 } } \left(\partial_{\mu}^{x_{2}} T\left(\wedge \varphi\left(x_{1}\right) \wedge \varphi\left(x_{2}\right)\right)\right.\right. \\
& \left.\left.\left.-T\left(\wedge \varphi\left(x_{1}\right) \wedge \varphi^{\mu}\left(x_{2}\right)\right)\right)\right]\right\}|k ; i n\rangle .
\end{aligned}
$$

Again one can prove that the last term under total derivative equals zero (if introduced packets). Continuing this inductive procedure we go to conclusion that all physical matrix elements of S-matrix in DKP theory coincide with those of KGF theory independent of character interaction (renormalizable type) if in both theories the LSZ asymptotic conditions (37) are implemented.

\section{Conclusions}

1) Starting from canonical approach to DKP theory interacting with quantized EM field and constructing the generating functional for GF of the theory we strictly proved total equivalence between physical matrix elements of $S$-matrix in DKP and KG theories and between many photons GF in both theories. The proof of equivalence between both theories have been carried out utilizing the Lehmann, Symanzik and Zimmermann reduction formalism [11].

We also proved the equivalence of the both theories, starting from Lagrangian approach to generating functional in DKP theory (see equation ( 16) without last term) and forgetting about constraints. 2) In principle, the DKP as well as KG theories are nonrenormalizable ones even for scalar particles due to the logarithmical divergence of one loop diagrams of scattering two particles with exchange of two photons [5]. As it is well known that KG theory becomes renormalizable if we introduce a self interaction term $\sim \lambda\left(\varphi^{*} \varphi\right)^{2}$. This problem can be solved in DKP theory in the same way: it is necessary to add to $\mathcal{L}$ in equation (1) terms

$$
\lambda(\bar{\psi} P \psi)^{2}=\lambda\left(\varphi^{*} \varphi\right)^{2},
$$

where $P=\mu \sqcap\left(\beta_{\mu}\right)^{2}$ is the projector on the scalar part of $\psi$-function; $P$ is pseudoscalar . 3) In the framework the same method (Sections II and III) formally it is possible to prove equivalence between DKP and Proca equation for spin one particles, destructing from nonrenormalizability of these theories. 4) We would like to stress that DKP theory until now did not find wider application although this theory has some advantages just due to the degeneration of $\beta_{\mu}$ matrices (one very simple to calculate trace that of) and due to minimal character of interaction with EM fields. One compares expressions for $S$-matrix in both theories:

$$
\begin{array}{r}
S_{D K P}=T \exp \left\{i \int e \bar{\psi} \beta_{\mu} A^{\mu} \psi d^{4} x\right\}, \\
S_{K G}=T \exp \left\{i \int i e \left(\varphi^{*} \partial_{\mu} \varphi-\partial_{\mu} \varphi^{*} \varphi\right.\right. \\
\left.\left.-e \varphi^{*} A_{\mu} \varphi\right) A^{\mu} d^{4} x\right\} .
\end{array}
$$

In the last case the interaction contains proportional terms to $e$ and $e^{2}$. Due to this in higher (two and more loops) approximations combinatorial coefficients given to order $e^{2}$ before having a complicated form.

5) About equivalence of DKP and KG for description of unstable particles we would like to note that if we can apply conception of asymptotic states to some such particle and utilize for physical matrix elements of $S$-matrix the same method which has been used in Sections II and III, then the proof of equivalence is obvious: for instance, for decay of $K_{l}-$ mesons we can calculate the imaginary part of the GF of $K_{l}-$ meson and get equivalence with exactness redefining the $\varphi$ component of DKP $\psi$ function: $i \varphi_{K G}=\varphi_{D K P} / m$, see equation (49).

\section{Acknowledgments}

We would like to thank I. V. Tyutin, who suggested to use reduction formalism of LSZ (see Section III) to proof the equivalence of both theories, and D. M. Gitman for stimulating criticism . V.Ya.F. thanks to FAPESP for support (grant number 98/06237-0) and RFFI for partial support (grant number 99-01-00376). B. M. P. thanks CNPq for partial support.

\section{References}

[1] G. Petiau, Acad. Roy. de Belg., A. Sci. Mem. Collect 16 (1936).

[2] R.Y. Duffin, Phys. Rev. 54, 1114 (1938).

[3] N. Kemmer. Proc. Roy. Soc. A 173 , 91 (1939). 
[4] H. Umezawa, Quantum Field Theory, North-Holland,1956.

[5] A.I. Akhiezer and V.B. Berestetski, Quantum Electrodynamics 2nd ed. Inter.,New York, 1965.

[6] T. Kinoshita, Prog. Theor. Phys. 5 , 473 (1950); T. Kinoshita and Y. Nambu, ibid. 5, 749 (1950).

[7] B.M. Pimentel and J.L. Tomazelli, Prog. Theor. Phys. 45, 1105 (1995).

[8] R.A. Krajcik and M.M. Nieto, Am. Journ. of Physics, 45, 818 (1974).

[9] V.L. Ginzburg, Quantum Field Theory and Quantum Statistics, Essays in Honor of the Sixtieth Birthday of E.S. Fradkin, Vol. 2 (1987) 15, IOP Publishing Ltd. 1987, Adam Hilger, Bristol.

[10] E. Fischbach, et al., Phys. Rev. Lett. 26, 1200 (1971).
[11] H. Lehmann, K. Symanzik and W. Zimmermann, Nuovo Cim. 1, 425 (1955).

[12] D.M. Gitman and I.V. Tyutin, Quantization of Fields with Constraints, Springer-Verlag, New York/Berlin, 1990.

[13] V. Ya. Fainberg and B. M. Pimentel, hep-th/9911215.

[14] P.A.M. Dirac, Proc. Roy. Soc. A 246, 326 (1958).

[15] V. Ya. Fainberg, Quantum Field Theory and Quantum Statistics, Essays in Honor of the Sixtieth Birthday of E.S. Fradkin, 1, 1 (1987), IOP Publishing Ltd. 1987, Adam Hilger, Bristol.

[16] L. Faddeev and A. Slanov, Gauge Fields, Introduction to Quantum Theory, The Benjamin Cummings Publishing Company, 1980. 